\title{
Relationship between leptin and insulin levels and homeostatic model assessment (HOMA) index in Spanish pubertal children
}

\author{
Stefanie Schoppen, Beatriz Cano, Iria de Oya, Laura López-Simón, Pia Riestra, Manuel de Oya \\ and Carmen Garcés \\ Lipid Research Laboratory, Fundación Jiménez Díaz, 28040 Madrid, Spain
}

Leptin plays a central role in the maintenance of energy balance ${ }^{(1)}$. Leptin concentrations have been shown to be directly proportional to the amount of body fat. However, it is well known that disruption of leptin signalling in the hypothalamus results in obesity. Hyperleptinaemia and/or leptin resistance may play an important role in insulin resistance by participating in its origin. The association between plasma leptin and insulin among adults has been examined ${ }^{(2)}$, but studies in pubertal children are scarce.

The aim of the present study was to determine the relationship between plasma leptin and insulin levels and HOMA index in pubertal Spanish boys and girls of normal weight (NW) and overweight (OW).

The study included 356 healthy schoolchildren (165 male and 191 female) from the Comunidad de Madrid, with a mean age of 13.7 years, ranging from 12-15 years, who participated in the follow up of a voluntary survey, the Four Provinces Study, a large-scale crosssectional study in Spain examining cardiovascular risk factors in four large metropolitan areas of the country ${ }^{(3)}$. The girls and boys were classified into NW and OW children according to Cole et al. ${ }^{(4)}$. Obese children were included in the OW group because of their low number. Fasting $(12 \mathrm{~h})$ venous blood samples were obtained by venipuncture in Vacutainer tubes. Once centrifuged, fractions were separated and frozen at $-70^{\circ} \mathrm{C}$. Plasma leptin was determined by ELISA using a commercially-available kit (EIA-2395; DRG Instruments $\mathrm{GmbH}$, Marburg, Germany). Serum insulin concentrations were measured by radioimmunoassay using a commercial kit (BI-Insulin IRMA; Bio-Rad Laboratories, Hercules, CA, USA). HOMA index was calculated (fasting insulin $(\mu \mathrm{U} / \mathrm{ml}) \times$ fasting glucose $(\mathrm{mmol} / \mathrm{l}) /$ 22.5).

Leptin levels were significantly higher in girls than in boys in NW and OW groups $(P<0.0001)$. Insulin levels and HOMA index were higher in NW girls than NW boys but levels were not different between OW girls and boys. Leptin levels were highly correlated with anthropometric variables in NW and OW girls, while in boys (NW and OW) correlations were weaker, being significant only with BMI. In these pubertal children plasma leptin levels were associated with insulin levels and HOMA index in girls and boys. The correlations were especially strong in girls.

\begin{tabular}{llccc}
\hline & \multicolumn{3}{c}{ Leptin } \\
\cline { 2 - 5 } & \multicolumn{2}{c}{ Girls $(n$ 191) } & \multicolumn{2}{c}{ Boys $(n$ 165) } \\
\cline { 2 - 5 } & NW $(n 140)$ & OW $(n$ 51) & NW $(n$ 107) & OW $(n$ 58) \\
\hline Weight & $0.394^{* *}$ & $0.404^{* *}$ & 0.042 & 0.205 \\
Height & 0.003 & 0.113 & -0.148 & -0.229 \\
BMI & $0.519^{* *}$ & $0.474^{* *}$ & $0.242^{*}$ & $0.453^{* *}$ \\
Insulin & $0.390^{* *}$ & 0.213 & $0.274^{* *}$ & 0.212 \\
HOMA & $0.413^{* *}$ & 0.276 & $0.280^{* *}$ & 0.253 \\
\hline$* P<0.05, * * P<0.01$ & & &
\end{tabular}

$* P<0.05, * * P<0.01$.

In conclusion, the results demonstrate a relationship between plasma leptin and insulin levels and HOMA index in pubertal children. Furthermore, the correlation between leptin levels and anthropometric data and insulin levels seems to present a gender dimorphism. Further studies in children should focus on the interaction between the extent of obesity, leptin and insulin levels and insulin resistance.

1. Banks WA, Farr SA \& Morley JE (2006) Physiol Behav 88, 244-248.

2. Lawlor DA, Smith GD, Kelly A, Sattar N \& Ebrahim S (2007) Obesity 15, 1694-1701.

3. Garcés C \& de Oya M (2007) Rev Esp Cardiol 60, 517-524.

4. Cole TJ, Bellizzi MC, Flegal KM \& Dietz WH (2000) Br Med J 320, 1240-1243. 